\title{
Orienting responses to a change in stimulus significance
}

\author{
R. L. RAY and J. F. PIROCH \\ University of South Florida, Tampa, Florida $\$ 9620$
}

\begin{abstract}
Female human subjects received either one, five, or nine tone-light compound stimulus presentations followed without interruption by presentations of tone alone. Task subjects were instructed to make a reaction time response to onset of the light stimulus. Control groups made no RT responses. The effect of omission of the light on the orienting response was evaluated by examining magnitude of GSRs to tone stimulus onset. In groups that performed the RT task, GSRs to stimulus change (compound to simple) were found to be a function of the number of trials prior to stimulus change. This was not the case when subjects had not performed the RT task. The RT task itself was demonstrated to affect GSR magnitude and frequency. It was concluded that the behavioral significance of the stimulus and the number of significant stimuli previously presented to the subjects directly affected GSR magnitude to stimulus change.
\end{abstract}

O'Gorman (1973) concluded from his literature review that orienting responses (OR) to stimulus change are consistently seen only with increases in stimulus intensity, or less consistently, with changes in modality. It is difficult, of course, to interpret in a simple manner increases in response magnitude produced by increased stimulus intensity or change in modality, since these changes may be due either to the simple dynamogenic effects of stimulus intensity or to the dishabituating effect of change itself. In an important exception to O'Gorman's generalization, Kimmel (1960) found that GSR ORs were produced by both increases and decreases in the intensity of an auditory stimulus and that the size of the OR varied directly with the amount of change. Kimmel's subjects received stimulus change, however, only after 20 presentations of the initial stimulus. It is possible that these conflicting findings result from the fact that ORs to intensity decreases do not appear until after a substantial number of habituation trials have first been presented. In a recent study, Edwards (1975) found that subjects made GSR ORs to a reduction in intensity of an auditory stimulus only after 15 or more presentations of this stimulus. Subjects who received only four or eight presentations of the stimulus before the reduction in intensity failed to show a GSR OR to change. Edwards noted that ORs to stimulus change were first produced at the point at which the habituation curve became asymptotic.

In order to determine whether Edward's finding is peculiar to stimulus intensity, the present study investigated the effect of change in another variable which behaves similarly to stimulus intensity: the performance of a reaction time (RT) task. Both increased stimulus intensity and the inclusion of an RT task increase

This research was supported in part by a University Council. Vellowship for the first author. The paper is sponsored by Dr. H. D. Kimmel, who takes full editorial responsibility for its contents.
GSR magnitude as well as retard habituation (Van Olst, 1971). If GSR magnitude increases when task demands are withdrawn and the stimulus significance is changed, further evidence, uncomplicated by possible dynamogenic effects, would be provided for the phenomenon of OR recovery to stimulus change.

\section{METHOD}

Subjects were 42 female students at the University of South Florida who volunteered to earn extra credit in psychology courses. The first 30 subjects were randomly assigned to one of three groups. The last 12 subjects were randomly assigned to one of two additional groups. Each subject was seated alone in an Audiometric Model 1200 soundproof room. Superex headphones were placed on the subject and zinc-zinc sulphate GSR electrodes in Plexiglas cups were attached to the palm and back of the nonpreferred hand. A push button was placed in the preferred hand of subjects in the first three groups. Instructions were given to the subject over an intercom, informing her that physiological responses to stimuli would be measured and that she was to avoid moving around or coughing. Subjects in Groups I, II, and III were also asked to push the RT button as quickly as possible when a light was presented. After the experimenter read the instructions, several minutes elapsed before stimuli were presented. The subjects were then presented with simultaneous compound stimuli consisting of a circular white light produced by a multiple stimulus projector and a $92-\mathrm{dB} 1,000-\mathrm{Hz}$ tone. The stimulus duration was $3 \mathrm{sec}$ and the intertrial interval was $15 \mathrm{sec}$. After one, five, or nine compound stimulus presentations, subjects in Groups I, II, and III, respectively, received further presentations of the $92-\mathrm{dB}$ tone only (i.e., the light was omitted). Subjects in Groups IV and $\mathrm{V}$ received one or nine compound stimulus presentations before change to tone only, but had no RT task. It should be remembered that subjects in Groups I, II, and III performed the RT task when compound stimuli were presented. The GSR was recorded using a Texas Instruments Company Rectiriter and a Bio-Physical GSR amplifier. Current density was $7 \mu \mathrm{amps} / \mathrm{cm}^{2}$ and the responses were transformed to units of change in log conductance.

\section{RESULTS}

Groups I, II, and III all showed a typical decline in GSR magnitude over trials (see Figure 1). An analysis 


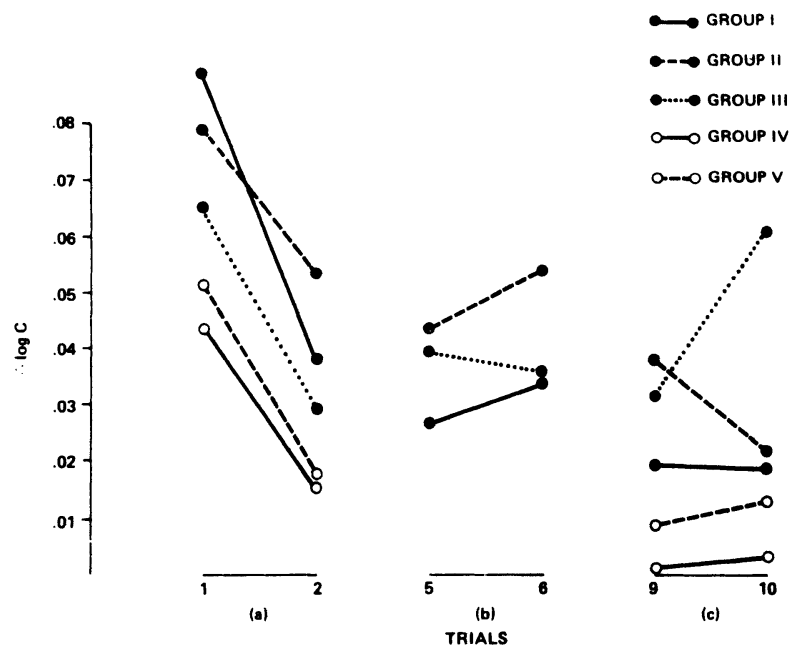

Figure 1. Change in GSR magnitude across Trials 1 and 2, 5 and 6 , and 9 and 10.

of variance showed that the GSR magnitude of the three groups did not significantly differ on Trial 1 . An analysis of GSR magnitude for the three groups over the first 10 trials showed that the effects of Trials $(F=11.91$, $\mathrm{df}=9 / 243)$ and the Groups by Trials interaction $(F=$ $2.74, \mathrm{df}=18 / 243$ ) were significant, but the effect of Groups was not $(F<1)$. Inspection of Figure 1a shows that Group I, which received stimulus change at Trials $1-2$, declined in response magnitude at this point, as did the other two groups. Figure $1 \mathrm{~b}$ shows that Group II, which received stimulus change at Trials 5-6, increased in response magnitude slightly at this point, as did Group I. Finally, Figure 1c shows that Group III, which received stimulus change at Trials 9-10, increased markedly in response magnitude at this point, while Groups I and II continued to decline. The increase in response magnitude seen here for Group III is obviously in the opposite direction to that predicted by a consideration of the dynamogenic properties of RT task (i.e., the intensity-like effect of the stimulus was $d i$ minished, not increased). In order to evaluate the effect of stimulus change, an analysis of GSR data from Trials 1-2, 5-6, and 9-10 was done for all three groups. The simple interaction of Trials by Groups was computed for each of these three pairs of trials. Only the simple interaction for the third pair of trials (9-10) was significant $(F=3.48, \mathrm{df}=2 / 27)$. This indicates that only after nine RT trials did the removal of task produce increased GSR magnitude. Groups IV and V showed a similar decline over trials in GSR magnitude. Also, the overall GSR magnitude was slightly greater for Group V than for Group IV. Analysis of variance showed that both the effects of Trials $(F=36.37, \mathrm{df}=9 / 90)$ and of Groups $(F=5.68, \mathrm{df}=1 / 10)$ were significant. The effect of stimulus change was evaluated for these two groups by computing the simple interaction of Trials by Groups for the two relevant pairs of trials, $1-2$ and $9-10$ (see Figures 1a and 1c, respectively). Neither of these simple interactions was significant. This finding indicates that the large increase in response size seen for Group III at Trial 10 was not simply due to omission of one element of a compound stimulus. Finally, in order to determine whether RT task produced its typical effect on the persistence of responses over trials, the frequencies of elicited GSRs during the first 10 trials for Groups I and III were compared to those of Groups IV and V. The mean frequency for Groups I and III combined was 9.45, while the mean frequency for Groups IV and V was 4.30. Analysis of variance showed that the above difference, the effect of RT vs. no RT task, was significant $(F=11.39$, df $=1 / 28)$. However, the effect of Number of trials before change, as well as the interaction of Number of trials before change and task was not significant.

\section{DISCUSSION}

The effect of change in stimulus significance on GSR OR magnitude was found to be a function of trials prior to stimulus change. This finding is similar to Edwards' (1975) finding for reduced stimulus intensity. However, it is uncertain whether this result depends on response magnitude having reached asymptote, as Edwards suggests. There was little difference between the response magnitudes of Group II on Trial 5 and Group III on Trial 9, but only Group III showed an effect of change. It will be remembered that Trials 5 and 9 are the trials immediately preceding stimulus change for Groups II and III. The results for the three task groups in the present study, as well as in Edwards' data, appear to support a Sokolovian approach to OR habituation and dishabituation (Sokolov, 1963). As the organism experiences more stimulations, a developing neuronal model of the stimulus becomes more complete. Since a more complete model allows a greater probability of detecting a mismatch between previous and present stimulations, the more experienced organism is more likely to respond with an OR to change. However, the results for the groups receiving change from compound to simple stimuli but never performing the RT task do not support a simple Sokolovian interpretation. Change from compound to simple stimuli did not produce an increase in response magnitude. This inconsistency probably is attributable to the saliency of change in the RT condition. A change from significant to nonsignificant stimuli may be more salient than the change from compound to simple stimuli (that is, omitting a task-relevant stimulus is more dishabituating than simply omitting a stimulus). The more salient change may then require a less completely formed neuronal model for a mismatch between previous and present stimulation to be detected. A possibly more parsimonious, but related, explanation would be that, since task subjects were required to attend to the stimulus, they were more surprised when the stimulus was changed from compound to simple.

However, it is interesting to note that many subjects (67\%) in the three RT task groups made an erroneous RT response on the first trial on which the element of the compound stimulus signal task was withdrawn. Many of these subjects reported to the experimenter that they had felt surprised or tricked when they responded incorrectly. However, this feeling of surprise was not translated into increased OR magnitude by subjects in Groups I and II. This finding, although incidental in nature, has obvious implications for OR theory in general, especially where it employs constructs such as stimulus novelty (Berlyne, 1960) and perceptual disparity (Grings, 1960). 


\section{REFERENCES}

Berlyne, D. E. Conflict, arousal, and curiosity. New York: McGraw-Hill, 1960.

EDWARDs, D. C. Stimulus intensity reduction following habituation. Psychophysiology, 1975, 12, 12-14.

Grings, W. W. Preparatory set variables related to classical conditioning of autonomic responses. Psychological Review, $1960,67,243-252$.

Kimmel, H. D. The relationship between direction and amount of stimulus change and amount of perceptual disparity response. Journal of Experimental Psychology, 1960, 59, 68-72.

O'Gorman, J. G. Change in stimulus conditions and the orienting response. Psychophysiology, 1973, 10, 465-470.

Soкolov, Y. N. Perception and the conditioned reflex. New York: MacMillan, 1963.

VAN OLST, E. H. The orienting reflex. Netherlands: Mouton, 1971.

(Received for publication April 7, 1976.) 